\title{
Abordajes de la temática migratoria en el Programa Jóvenes y Memoria
}

\author{
Rocío de la Canal \\ Universidad Nacional de Mar del Plata \\ @ [ rociodelacanal@gmail.com ]
}

DOI: http://dx.doi.org/10.19137/huellas-2017-2106

a temática migratoria y más recientemente las movilidades en todo
el mundo, presentan tantas perspectivas para su abordaje como estu-
diosos dedicados a la interpretación de las mismas. Es necesario tener en
cuenta varios aspectos para encarar un análisis en profundidad pero prin-
cipalmente indagar sobre las motivaciones y situaciones que enfrentan los
migrantes en sus lugares de origen, y que se presentan como características
inherentes.

Las condiciones de vulnerabilidad entendidas como la falta de empleo digno y regulado, de servicios sociales tales como el acceso a la salud, la vivienda, la educación, como también los enfrentamientos entre diferentes grupos, la violencia armada, la marginación y la falta de integración e inclusión constituyen estas motivaciones; sabemos que estas condiciones deben ser tratadas por los actores que detentan el poder en cada uno de los Estados, sin embargo pocos son los que plantean soluciones a las problemáticas sociales, y que además abarcan un número importante de individuos, pero que a pesar de ello, no parece representar un interés para los gobiernos.

Este cúmulo de situaciones que pueden ser enmarcadas bajo el concepto de violencia, formará parte en las decisiones de trasladarse a un lugar diferente al de origen. Sin embargo cabe resaltar, que no todas las personas que sufren violencia, tienen la posibilidad de moverse, ya que entran en juego otra serie de factores tales como la capacidad monetaria para encarar el desplazamiento, la relación o no con paisanos que previamente se desplazaron y que de algún modo pudieran facilitar la llegada a través de redes y cadenas migratorias, acogida e integración del migrante y la cuestión documentaria que plantea una problemática en nuestros tiempos de vital relevancia. 
Es necesario a su vez, tener en cuenta que la regularidad o irregularidad de documentos trae aparejados diversos conflictos, aunque en nuestro país, no ha representado situaciones de gravedad ya que se han mantenido por lo general políticas de apertura inmigratoria y acogida, pero que en algunos períodos históricos, las políticas migratorias argentinas, sufrieron altibajos como durante el desarrollo de los gobiernos militares de los setentas y actualmente bajo el gobierno de turno.

Una de las entidades que se ha dedicado a la protección de los Derechos de las personas, entre ellos de los migrantes llegados a la Argentina, es la Comisión Provincial por la Memoria (CPM), institución no gubernamental autónoma y autárquica, que se integra por diversos organismos de Derechos Humanos, intelectuales de distintas disciplinas, funcionarios, universitarios y judiciales, como también artistas, representantes religiosos de múltiples credos, legisladores y sindicalistas, comprometidos con la ardua tarea de mantener la memoria colectiva para pensar el presente y el futuro de las sociedades y resguardar los derechos de todas las personas, con especial énfasis en la profundización de las memorias relacionadas con el terrorismo de Estado y la promoción y defensa de los Derechos Humanos y el bien común.

A partir del 2002, en la CPM comienza a funcionar un programa dedicado a las juventudes escolarizadas en el ámbito público y privado, el Programa Jóvenes y Memoria, con el fin de abordar temáticas que estuvieran latentes o manifiestas en la historia de los jóvenes que no vivieron la época del proceso, pero que de una manera u otra se ven afectados sin dudas, por las secuelas dejadas en la sociedad argentina y las derivaciones de exclusión, discriminación y violencias que quedan como resabios de aquella época.

El Programa se pone en funcionamiento mediante una serie de pautas con el fin de organizar el trabajo colectivo, siendo el alcance del mismo en un principio solo en el territorio de la Provincia de Buenos Aires, pero que posteriormente se ampliará abarcando otras provincias del país, estos grupos son coordinados por otras entidades relacionadas con la CPM, pero de manera independiente.

Las secuencias de trabajo se dan a partir de convocatorias abiertas a entidades educativas públicas y privadas, organizaciones civiles, barriales y otras asociaciones que se encuentren interesadas en participar. Una vez definidas las temáticas a trabajar entre los grupos, alumnos y docentes participantes $^{1}$, se ordenan en diferentes ejes propuestos por el Programa pero

1 Son alumnos de los cursos superiores de educación secundaria y media, de sexto año. 
de elección libre, y posteriormente se organizan a lo largo del ciclo lectivo talleres y encuentros regionales, dedicados a los docentes y participantes.

Los talleres son coordinados por equipos de voluntarios que participan con la CPM y el Programa, se realizan aportes y discusiones, intercambios de ideas, se desarrollan encuentros guiados en el trayecto de investigación, dirigidos tanto a los docentes como a los equipos integrales, posteriormente se evalúan los trabajos. Finalmente se realiza un encuentro en la ciudad de Chapadmalal, de periodicidad anual donde se exponen todos los trabajos realizados por los chicos y las organizaciones que hubieran participado, las producciones se presentan en varios soportes, tales como obras de teatro, música, murgas, producción audiovisual, literatura, entre otros buscando como fin el intercambio y enriquecimiento del trabajo colectivo.

La Comisión posee un catálogo general, y específicamente uno correspondiente al Programa Jóvenes y Memoria que consta de 3346 producciones al momento (año 2002 y 2014). A partir de la realización de una práctica profesional, se llevó a cabo el análisis de producciones que abordaban la temática de la migración en la Argentina, dando como resultado de la búsqueda en catálogo de 28 trabajos, todos ellos pertenecientes a escuelas de nivel medio. De estas producciones se seleccionaron doce que se consideraron representativos del grupo de los veintiocho trabajos que trataban el tema ${ }^{2}$. La búsqueda en el catálogo, plantea diferentes aportes para el investigador, colabora en la búsqueda de archivos, presenta una guía ordenada para quién ejerce la investigación, permite realizar comparaciones, encontrando semejanzas y similitudes por año de producción y entre períodos, el catálogo es público y abierto, y se encuentra en línea un resumen de cada producción.

El abordaje teórico de las producciones fue realizado desde diversas perspectivas disciplinares como sociológicas, antropológicas y geográficas, ya se consideró que el estudio de las migraciones debe realizarse con una perspectiva multidisciplinar por la complejización que conllevan los procesos migratorios. A partir de la revisión bibliográfica, se recuperaron términos y conceptos que se adecuaron al análisis de las producciones y

2 Una versión preliminar de este trabajo fue presentada en el marco de las XVIII Jornadas de Investigación, organizadas por el Centro de Investigaciones Geográficas y el Departamento de Geografía de la Facultad de Humanidades y Ciencias de la Educación en la UNLP. Tuvo como objetivo dar a conocer la experiencia de práctica profesional, en el marco de las horas de investigación que requiere el Plan de Licenciatura en Geografía para la obtención del grado, y que fueron realizadas en la Comisión Provincial por la Memoria entre los meses de agosto y diciembre de 2015. La metodología se orientó a la búsqueda en catálogo, el relevamiento bibliográfico relacionados con la temática migratoria y de Derechos Humanos y asistencia a talleres brindados en el marco del Programa. 
permitieron darle un marco teórico al trabajo de los alumnos, para desentramar las visiones que adoptaron para cada producción. Entre estos grupos de conceptos se tomaron la segregación espacial, marginación, estigmatización, discriminación y xenofobia; redes y cadenas migratorias e imaginarios colectivos; cada uno de ellos será abordado más adelante en relación a las tres producciones analizadas.

El análisis de tres trabajos considerados los más representativos del conjunto, se indagaron con el fin de obtener lo más profundamente posible, la visión de las juventudes participantes; el primero de ellos "No te enseño mi lengua, no quiero que te carguen", fue realizado en Ezpeleta, catalogado en el eje temático Género y Diversidades -Identidades; se aborda la cuestión de la identidad a través del uso de la lengua materna, en el que claramente se encontraron que miembros de la escuela, porteros y auxiliares, intentaban ocultar su origen y lugar de procedencia, hablando solo la lengua castellana. En las entrevistas realizadas por los chicos, pudieron ver que el uso de la lengua nativa, era tomada por los actores como elemento de discriminación y exclusión, y que intentaban esconderse del resto del equipo académico, e incluso de los alumnos de la institución quedando el uso del quechua, aymara y guaraní reservado al ámbito familiar y de las amistades.

Los inmigrantes entrevistados, provienen de países limítrofes a la Argentina, principalmente Bolivia y Paraguay, siendo Ezpeleta una ciudad con un número importante de residentes originarios de estos países, quienes arribaron en las últimas décadas del siglo veinte, principalmente en la década del '70. La paradoja en este trabajo, es que tanto los clubes deportivos como la comunidad educativa y el barrio, promueven actividades de respeto por la diversidad y valoración de las culturas, fomentando la integración de todos mediante la organización de festivales, encuentros de música y desfiles, por lo que se halla una contradicción en este caso, entre el deseo de la sociedad en la cual están insertos los migrantes, y la percepción que ellos tienen de la sociedad en la que viven que sin lugar a dudas es tan negativa, que prefieren negar su identidad.

Otro de los trabajos "Diferentes pero iguales" de Villa Luro, Partido de Villarino en la Provincia de Buenos Aires, catalogado en el eje Género y Diversidades, se abordaron las cuestiones relacionadas con el ámbito escolar y el barrio, indagando sobre los derechos de las personas y qué es ser diferentes pero iguales. Se realizaron recorridos por los barrios aledaños al colegio, donde al igual que en Ezpeleta, la mayoría de sus habitantes provienen de países limítrofes a la Argentina. Se llevaron a cabo entrevistas a miembros del club barrial quienes manifestaron la necesidad y el compro- 
misos de estas entidades, como también las instituciones del ámbito educativo en la integración de los migrantes, pero principalmente la importancia de integrar las juventudes e infancias, que puedan crecer en lugares donde no son discriminados y donde a pesar de que todos somos diferentes por muchas características que nos son propias de cada uno, todos tenemos los mismos derechos y por tanto nos corresponden las mismas oportunidades.

La última de las producciones tomadas para esta reflexión es "Inmigración, identidad y discriminación", de la localidad de Los Porteños, Partido de La Plata en la Provincia de Buenos Aires, se analizó la problemática de la explotación laboral de migrantes bolivianos, trabajadores rurales en el cinturón hortícola de La Plata. La realización de los chicos apunta a mostrar como los propios migrantes bolivianos arribados al menos diez años antes que los migrantes bolivianos empleados, ejercen la explotación, exclusión e incluso chantaje de sus compatriotas, presumiendo un status diferencial y no solo tomando ventaja abusiva del trabajo ajeno, sino también otorgándoles condiciones de vida precarias a los recién llegados y sus familias.

Por otra parte, los hijos de quinteros son discriminados en el ámbito escolar, debido al gran ausentismo que provoca el trabajar con sus padres y familiares en la cosecha, creando un ambiente de marginados y autoexcluidos como consecuencia de esta situación de desigualdad. Los chicos han mostrado mediante entrevistas, el sufrimiento de los inmigrantes bolivianos residentes en Los Porteños, el maltrato diario y la desvalorización de sus trabajo, quienes manifiestan que el ser pobres es lo que marca las diferencias y que esto los deja al desamparo, que no tienen derecho al reclamo ni la protestan ni por aquello que consideran y es justo para todos.

La desigualdad que se genera entre el empleado y el empleador plantea un rezago en materia no solo de derechos humanos, sino también del rol que juegan las entidades gubernamentales de la Ciudad de La Plata, que conociendo la situación, es cómplice del accionar del explotador boliviano sobre sus compatriotas.

Finalmente, estos migrantes que arribaron a la Argentina, un primer grupo en la década de los '70s, y otro flujo fuerte en volumen y dinámica en la década de los '90s. En el primer caso, es coincidente con el fomento de la migración y de inversiones en la Argentina por parte de los gobiernos de facto que a sus fines, no resultó como esperaban, ya que la inmigración que arribo al país era la indeseada, esto significa que los sujetos que ingresaron al país procedían de países limítrofes a la Argentina, muchos de zonas campesinas, y de grupos étnicos como guaraníes, quechuas y aimaras, muy alejado del prototipo esperado por la "Argentina blanca". Aquí, queda claramente identificadas las relaciones de poder, la explotación y el 
no derecho a una vivienda digna, la marginación de sus paisanos y su auto marginación, la imposibilidad de acceder a la justicia y la educación por ser pobres e "ignorantes".

Los estereotipos sobre las poblaciones migrantes limítrofes fueron recurrentes en los ámbitos laborales, educativos y sanitarios, sin embargo en las producciones, no se muestra una impronta discriminatoria tan pronunciada o visible durante los ' 70 , época violenta y de persecuciones conocidas por todos, como si se visibilizó más en los noventa. Debemos considerar que los discursos políticos ${ }^{3}$ de la época, buscaron una explicación que diera fundamentación a las altas tasas de desempleo, la pobreza y la desigualdad social que caracterizó al periodo, a partir de las condiciones que se fueron generando como consecuencia de la adopción por parte del gobierno de Carlos Menen, de políticas económicas neoliberales. Los inmigrantes limítrofes, funcionaron como chivo expiatorio, siendo acusados de robar el trabajo ajeno, y como únicos causantes del malestar de la Argentina. Tanto la problemática migratoria como el ejercicio de los derechos son cuestiones fundamentales para ser abordado no solo como temáticas sociales sino también como problemáticas. Desmitificar las creencias acerca de los migrantes indeseables es fundamental en la construcción de horizontalidades y en la integración de la comunidad; la falta de políticas sociales dedicadas a estas problemáticas no deben ser un detalle menor.

\section{Referencias bibliográficas}

DOMENECH, E. (2009). La "nueva política migratoria” en la Argentina: las paradojas del programa "Patria Grande". Publicado originalmente en francés en: Problemes d'Amerique Latine, París. Francia, núm. 75, pp. 37-59. Publicado en Argentina en el libro: Migración y política: el Estado interrogado.

ELIAS, N. (2003) Ensayo acerca de las relaciones entre establecidos y forasteros. En: Reis Revista Española de Investigaciones sociológicas, núm. 104,. Madrid, España. Pp. 219251 .
GRIMSON, A. (2011). Doce equívocos sobre las migraciones. En: Revista Nueva Sociedad $\mathrm{N}^{\circ}$ 233, mayo-junio ( $\sin$ información de páginas).

ROMERO, L. A. (1994) Breve Historia Contemporánea de la Argentina. Buenos Aires, Argentina.

SEGURA, R. (2006). Segregación residencial, fronteras urbanas y movilidad territorial. Un acercamiento etnográfico. En: IDES $\mathrm{N}^{\circ} 9$. Buenos Aires, Argentina.

3 Los discursos pronunciados por el entonces gobernador de la Provincia Eduardo Duhalde y el entonces Ministro de Economía Domingo Cavallo. 